\title{
Evaluation of energy barriers for topological transitions of Si self-interstitial clusters by classical molecular dynamics and the kinetic activation- relaxation technique
}

\author{
Pedro López ${ }^{1}$, D. C. Ruiz², I. Santos ${ }^{1}$, M. Aboy ${ }^{1}$, L. A. Marqués ${ }^{1}$, M. Trochet ${ }^{3}$, N. Mousseau ${ }^{3}$, and L. Pelaz ${ }^{1}$ \\ ${ }^{1}$ Departamento de Electricidad y Electrónica, Universidad de Valladolid, ETSI Telecomunicación, Paseo de Belén 15, 47011 \\ Valladolid, Spain \\ ${ }^{2}$ Department of Information Technology and Electrical Engineering, ETH Zürich, Gloriastrasse 35, 8092 Zürich, Switzerland \\ ${ }^{3}$ Département de Physique and Regroupement québécois sur les matériaux de pointe, Université de Montréal, C.P. 6128, succ. \\ Centre-ville, Montréal (Québec) H3C 3J7, Canada
}

\begin{abstract}
The modeling of self-interstitial defects evolution is key for process and device optimization. For a self-interstitial cluster of a given size, several configurations or topologies exist, but conventional models assume that the minimum energy one is instantaneously reached. The existence of significant energy barriers for configurational transitions may change the picture of defect evolution in non-equilibrium processes (such as ion implantation), and contribute to explain anomalous defect observations. In this work, we present a method to determine the energy barriers for topological transitions among small self-interstitial defects, which is applied to characterize the Si self-interstitial and the di-interstitial cluster.
\end{abstract}

Keywords—silicon; self-interstitial cluster; configurational transition; energy barrier

\section{INTRODUCTION}

Self-interstitial defects are commonly formed in Si as a consequence of fabrication processes, and may range from small selfinterstitial clusters (I-clusters) to extended defects, such as rod-like defects and dislocation loops. For I-clusters several configurations have been reported, each one characterized by a specific topology and a formation enthalpy [1]. Traditional defect evolution models only consider the minimum energy configuration for each cluster size, by assuming that there are no barriers among them. These models successfully describe defect kinetics [2], but they fail to explain some experimental observations, like the growth of dislocation loops in shallow Ge implanted Si, without the previous formation of rod-like defects [3].

The relevance of higher energy I-clusters configurations depends to a large extent on the existence and height of energy barriers for configurational transitions. The Nudged Elastic Band Method (NEBM) can be used to calculate energy barriers in a static way, by considering the initial, final, and at least one intermediate position of all moving atoms. As a predefined atomic position along the pathway for a configurational transition is considered, calculated energy barriers may be overestimated, if the pathway followed is not the lowest energy one. Classical molecular dynamics (CMD) uses a semi-empirical potential to simulate the vibration and movements of atoms, can provide the energetic and topological description of I-clusters, and simulate their dynamics. However, the determination of energy barriers for configurational transitions with CMD may be very time consuming, as these transitions are usually infrequent events compared to the fast vibrational movement of atoms. 
In this paper, we describe a method to calculate energy barriers for configurational transitions between small self-interstitial defects, by using the kinetic Activation-Relaxation technique (kART) [4, 5]. With this technique, no predefined pathways for transitions are considered, and large energy barriers can be reached, since the vibration of atoms is not simulated.

\section{SIMULATION SCHEME}

The kART is used to determine the energy barriers between the main configurations of the Si self-interstitial and the diinterstitial cluster (I2 cluster).

\section{A. Kinetic Activation-Relaxation Technique}

The kinetic Activation-Relaxation Technique is an off-lattice self-learning kinetic Monte Carlo (kMC) method which identifies and evaluates activation barriers, and provides a topological description of events. From a local energy minimum, associated to a particular atomic configuration, the kART identifies the saddle points that lead to other local minima in an open-ended approach (Fig. 1). This is done by applying a local deformation to a randomly selected atom and its neighbors, and by pushing the system to go through a saddle point and to reach a new energy minimum. As an open-ended method, no predefined pathways for transitions are considered, contrary to other techniques such as the NEBM. Energetics of minima and saddle points are derived from a semiempirical potential. Transitions between neighboring local minima through a saddle point are classified according to their activation energy, and are chosen by a kMC scheme. Events found are stored in a catalog and barriers are fully relaxed before each kMC step to ensure that all elastic deformations are considered.

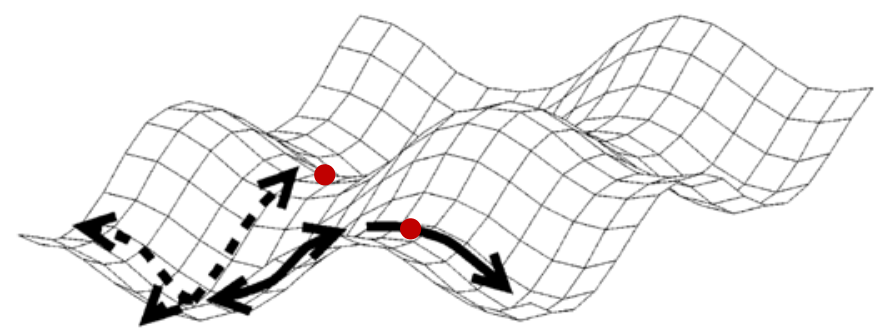

Fig. 1. Transitions between energy minima through saddle points (red dots) (modified from [5]).

As the kART classifies available events for leaving a local minimum according to their activation energy (i.e. the energy of the saddle point with respect to its initial local minimum), those events with low energy barriers have a high probability of being selected by the kMC algorithm. When several neighboring local minima are connected by low energy events, the system may continuously evolve from one to another of these minima. Additionally, if the events for leaving a local minimum have a low reverse barrier, when one of these events is performed the system will easily come back to the starting minimum. Both situations may cause the system to be trapped in a few minima, preventing that higher energy states are reached.

Increasing the simulation temperature may favor that more energetic states are visited, but most computational time is still devoted to low energy events. We use the bac-MRM method [6] to solve analytically the residence time between these low-energy states (with energy barriers below a predefined value, the basin-energy threshold), allowing us to focus on the evolution driven by higher-energy mechanisms.

The kART relies on the accuracy of the semi-empirical potential used for the energetic characterization of local minima and saddle points. Several studies have combined the kART and the Stillinger-Weber (SW) potential [7] for the analysis of defects in Si 
[8, 9], but there are no references so far of the use of the kART with the Tersoff potential. In order to provide a comparison of both potentials through the kART, we have studied the Si self-interstitial with the SW and the Tersoff potential in its third parametrization [10].

\section{B. Simulation procedure}

This work is focused on the analysis of configurational transitions of Si self-interstitials and di-interstitial clusters. The Si selfinterstitial configurations included in this study were taken from those reported in the literature [11]. For the I2 clusters, we have used CMD simulations to identify and characterize the most common configurations [12]. CMD simulations were performed with the parallel code LAMMPS [13] and the Tersoff potential in its third parametrization. Two self-interstitials were introduced in a crystalline Si cell close to each other, and were annealed at $1200 \mathrm{~K}$ for $25 \mathrm{~ns}$. The resulting I2 clusters were then characterized at 0 $\mathrm{K}$ and $0 \mathrm{GPa}$.

The minimum energy configurations of the Si self-interstitial and the I2 clusters were simulated with the kART for 1000 kMC steps. The energetics of each kMC step was determined through the coupling of the kART with LAMMPS and the Tersoff potential, and also with the SW potential for the analysis of the Si self-interstitial. Simulation temperature was set at $500 \mathrm{~K}$ and 50 attempts were used to ensure that most possible events from each configuration were identified.

Most of the configurations, or local energy minima, visited during the simulations are similar, with only small variations on the energy or on the position of atoms with respect to the most stable configuration. In order to make a significant study of the observed configurational transitions, we have grouped configurations in families, based on geometrical and energetic criteria. This is illustrated in Fig. 2, in which starting from a dumbbell configuration of a Si self-interstitial (orange diamonds), other local minima with similar characteristics are visited, until a significant change in energy and geometry is observed (steps 4-7). The selfinterstitial has underwent a topological transition from dumbbell-like to tetrahedral-like configurations (red circles). The aim of this work is to calculate the energy barriers for the transitions between configurational families, which are defined by the energy of the highest saddle point that has been overcome (dashed circle in Fig. 2).

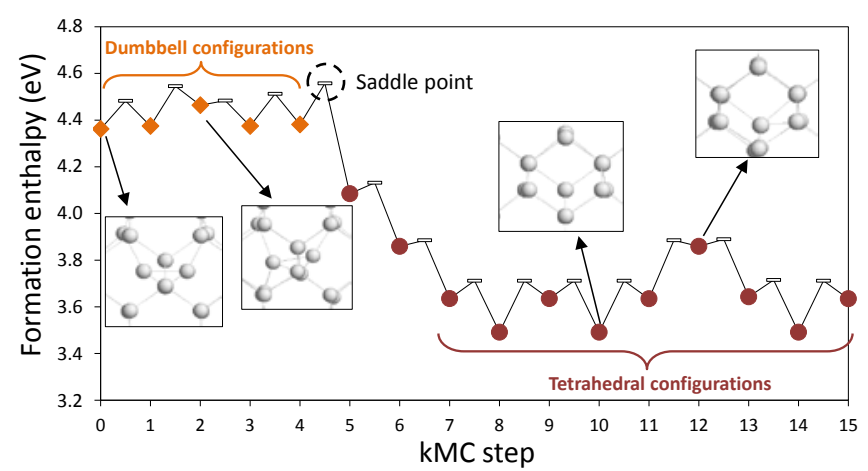

Fig. 2. Energy states from a kART simulation with the Tersoff potential in which a transition between two familes of self-interstital configurations has ocurred (dumbbel in orange diamonds, and tetrahedral in red circles). The dashed circle shows the saddle point that defines the energy barrier for this transition. 


\section{RESULTS}

\section{A. Si self-interstitial}

The main topologies of the Si self-interstitial are the tetrahedral (T), the dumbbell (D) and the extended (E) configurations [11]. Our calculations with the SW potential indicate that the ground state of the Si self-interstitial is the E configuration (3.68 eV), and the $\mathrm{D}$ and $\mathrm{T}$ configurations have a larger energy ( $4.43 \mathrm{eV}$ and $4.95 \mathrm{eV}$, respectively). These values are similar to those reported in literature [11]. As can be seen in Fig. 3, the T configuration is unstable with this potential, and it easily evolves to the extended selfinterstitial, from which the D configuration can be reached. Direct transitions are also observed between E configurations (steps 13), or D configurations (steps 9-13), in this latter case with two different energy barriers.

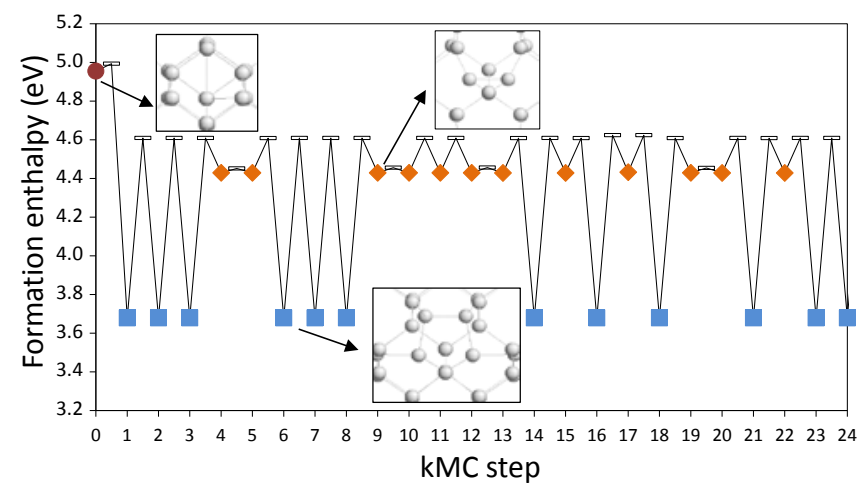

Fig. 3. Energies of the local minima and saddle points (open rectangles) of the first kMC steps of a simulation with the SW potential starting from the tetrahedral Si self-interstital. The insets show the geometriy of the associated configuration families, indicated by different colors and symbols: tetrahedral (red circles), dumbbell (orange diamonds), extended (blue squares).

The configurational transitions observed for the Si self-interstitial are summarized in Fig. 4. Some transitions may occur through a low (solid line) or a high energy (dashed line) pathway, as in the case of the E configuration, with barriers of $0.77 \mathrm{eV}$ and $0.94 \mathrm{eV}$ to transform into the dumbbell configuration. The more energetic D self-interstitial, and especially the $\mathrm{T}$ configuration, face lower barriers. Our results are coincident with previous reports with this technique [8, 9]. With the SW potential, configurational transitions are frequent, the system can easily leave the ground state, and it is not trapped in low energy events.

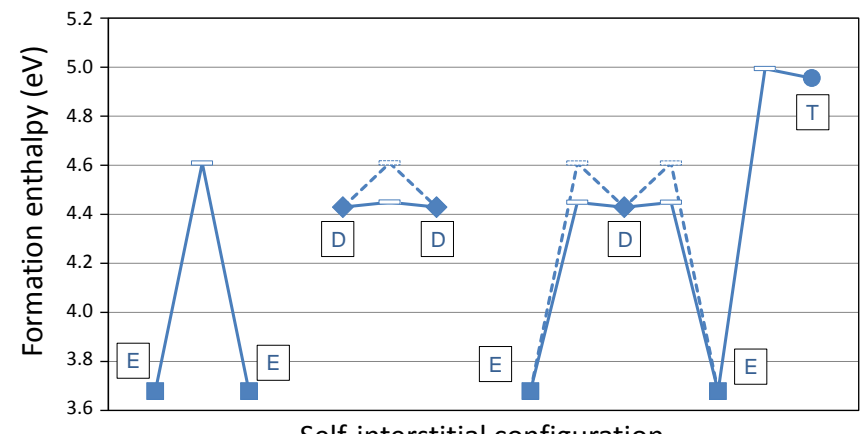

Self-interstitial configuration

Fig. 4. Formation enthalpies and saddle points (open rectangles) for the configurational transitions of the Si self-interstitial with the SW potential. When there are two saddle points for the same transition, solid and dashed lines indicate the lower and higher energy pathways, respectively. 
We have also studied the Si self-interstitial with the Tersoff potential, with the same parameters for the kART that we used with the SW potential. Starting from the $\mathrm{T}$ configuration (the ground state for the Tersoff potential at low temperatures) no configurational transitions are observed during the $1000 \mathrm{kMC}$ steps of the simulation. All visited states correspond to slight variations of the tetrahedral self-interstitial (similar to those reported in Fig. 2 with red circles), but no transitions to the D or E configurations took place.

Configurational transitions are not as straightforward with the Tersoff potential as those observed with the SW potential. In fact, several local energy minima correspond to the same family of self-interstitial configurations, with little variations on energy and geometry, and the system must climb to the highest energy states of a given family before a configurational transition occurs. The energy landscape resulting from the Tersoff potential seems to be somehow rough, which implies that there are several neighboring local energy minima that can be reached from the lowest energy minimum. These minima are usually shallow, i.e. they have low energy barriers to come back to the initial configuration. As we explained in Section II, this situation holds the system and prevents it from evolving to other configurations.

To overcome this limitation, we forced the kART to visit higher energy states by increasing the basin-energy threshold, and we run simulations starting from the D and E self-interstitials. The formation enthalpies and energy barriers we obtained are reported in Fig. 5, together with other data from the literature calculated by CMD [11] and the NEBM [14]. In our simulations the D and E configurations are less energetic than the CMD calculations. The transformation between the $\mathrm{T}$ and E self-interstitials always occurred through the dumbbell configuration, and no direct transition between T and E was observed. Energy barriers calculated with the NEBM are larger than those obtained with the ART. As the NEBM requires to assume in advance some intermediate positions of atoms for a configurational transition, energy barriers are usually overestimated. For the E configuration, the large energy barrier obtained with the NEBM corresponds to a direct transition from E to T configurations, which is a higher energy pathway that the transition through the dumbbell self-interstitial we observed with the kART.

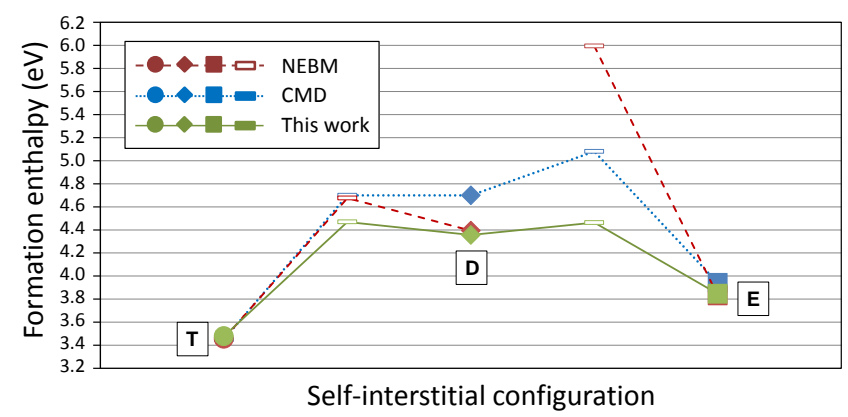

Fig. 5. Formation enthalpies and saddle points (open rectangles) for the Si self-interstial with the Tersoff potential (green symbols and solid lines). Data from the NEBM (from [14]) are plotted with red symbols and dashed lines, and from CMD simulations (from [11]) with blue symbols and dotted lines.

\section{B. Energy barriers for the configurational transitions of the di-interstitial cluster}

As defect size increases there is a larger variability of atoms positions, which hinders the identification of topologies. Our CMD simulations revealed that most configurations of the di-interstitial cluster could be classified either as a compact, or as a chainlike elongated structure, shown in the insets of Fig. 6. The compact structure is slightly more stable than the chainlike one, with formation enthalpies for the ground state of 6.40 and $6.43 \mathrm{eV}$, respectively. We performed 7 different simulations with the kART and the Tersoff potential for the I2 clusters ( 2 starting from the compact I2, and 5 from the chainlike one) at $500 \mathrm{~K}$, with an energy 
threshold of $0.3 \mathrm{eV}$ to avoid low energy events. We found 14 different compact configurations and 9 chainlike ones, whose formation enthalpies and relative frequencies are reported in Fig. 6.

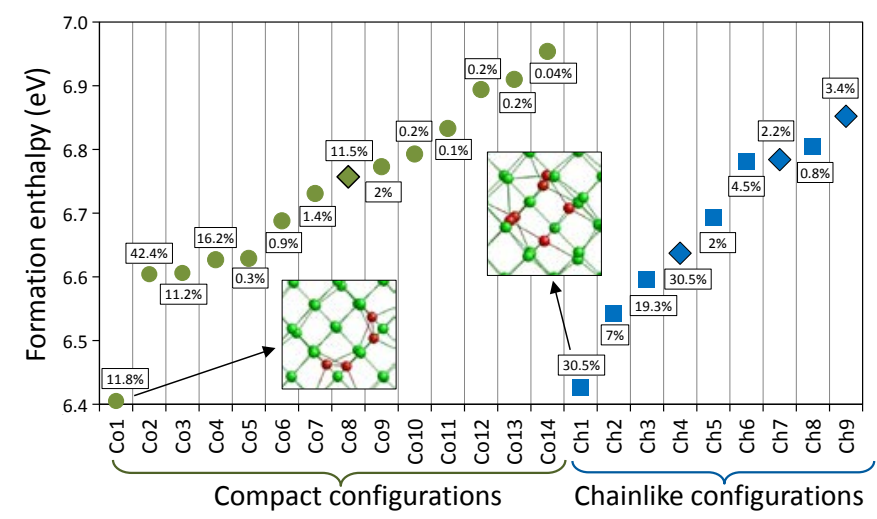

Fig. 6. Formation enthalpies and relative frequency of energy minima for the compact (green circles) and chainlike (blue squares) configurational families of I2 clusters. Diamonds indicate those configturations from which transitions were observed. The insets show the topologies of minimum energy configurations (displaced atoms are plotted in red).

Topological transitions between the two families were observed only in simulations starting from the chainlike I2 cluster. Despite the large variety of configurations, only four of them were involved in topological transitions (diamonds in Fig. 6). Transitions were relatively frequent (around 50 were observed), and occurred through different pathways, whose energy barriers are indicated in Fig. 7 as empty rectangles.

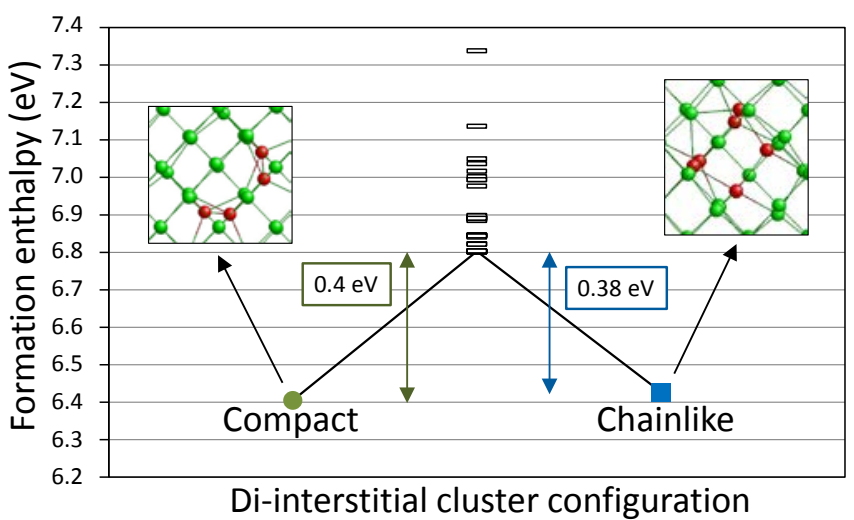

Fig. 7. Formation enthalpies of mininum energy configurations for the compact and chainlike families of I2 clusters, and energy barriers for toplogical transitions. The line represents the lowest energy pathway for the transition, with the associated energy barriers. The insets show the topologies of minimum energy configurations (displaced atoms are plotted in red).

The line in Fig. 7 shows the lowest energy barrier reported, $0.4 \mathrm{eV}$ and $0.38 \mathrm{eV}$, for direct and reverse transitions from compact to chainlike I2 clusters, respectively. These barriers, remarkably lower than the $2.2 \mathrm{eV}$ value obtained with the NEBM [14], are consistent with the low migration energy reported for the I2 cluster $[9,15]$. 


\section{CONCLUSSIONS}

We have presented a method for evaluating the energy barriers for topological transitions of small Si self-interstitial defects, by using the kinetic Activation-Relaxation technique. With this technique, no predefined pathways for transitions are considered, and large energy barriers can be overcome, since the vibration of atoms is not simulated. We have observed that, with the Tersoff potential, configurational transitions for the Si self-interstitial are not as straightforward as with the SW potential. The Tersoff potential seems to provide a rough description of the energy landscape, with many shallow energy minima associated to slight variations of a stable configuration, which hinders that other topologies with a larger energy can be reached. For the di-interstitial cluster classical molecular dynamics simulations were used to determine the most stable configurations, the so-called compact and chainlike structures. Transitions between these topologies were frequently observed in the kART simulations, and energy barriers of $0.38-0.4 \mathrm{eV}$ were calculated. The determination of energy barriers for topological transitions can be easily applied to other sizes and types of defects, and to different materials, and may contribute to the development of more detailed defect evolution models.

\section{ACKNOWLEDGMENT}

This work has been funded by the Spanish Government under project TEC2014-60694-P, and the JCyL Consejería de Educación y Cultura under project VA331U14.

\section{REFERENCES}

[1] S. Lee, and G. S. Hwang, Phys. Rev. B, vol. 78, pp. 045204, 2008

[2] N. E. B. Cowern et al., Phys. Rev. Lett., vol. 82, pp. 4460-4463, 1999.

[3] F. Gutierrez, K. S. Jones, and D. F. Downey, Mat. Res. Soc. Symp. Proc., vol. 669 (J5.11), pp. 1-5, 2001.

[4] F. El-Mellouhi, N. Mousseau, and L. J. Lewis, Phys. Rev. B, vol. 78, pp. 153202, 2008.

[5] G. T. Barkema, and N. Mousseau, Phys. Rev. Lett., vol. 77(21), pp. 4358, 1996.

[6] L. K. Béland, P. Brommer, F. El-Mellouhi, J.-F. Joly, and N. Mousseau, Phys. Rev. E, vol. 84, pp. 046704, 2011.

[7] F. H. Stillinger, and T. A. Weber, Phys. Rev. B, vol. 31, pp. 5262, 1985.

[8] P. Ganster, L. K. Béland, and N. Mousseau, Phys. Rev. B, vol. 86, pp. 075408, 2012.

[9] M. Trochet, L. K. Béland, J. -F. Joly, P. Brommer, and N. Mousseau, Phys. Rev. B, vol. 91, pp. 224106, 2015.

[10] J. Tersoff, Phys. Rev. B, vol. 38, pp. 9902, 1988.

[11] L. A. Marqués, L. Pelaz, P. Castrillo, and J. Barbolla, Phys. Rev. B, vol. 71, pp. 085204, 2005.

[12] I. Santos, M. Aboy, P. López, L. A. Marqués, and L. Pelaz, J. Phys. D: Appl. Phys., vol. 49, pp. 075109, 2016.

[13] http://lammps.sandia.gov.

[14] I. Santos, and S. Retuerto, private communication, 2015.

[15] G.H. Gilmer, T. Diaz de la Rubia, D. Stock, and M. Jaraiz, Nucl. Instrum. Methods, Phys. Res. Sect. B, vol. 102, pp. $247,1995$. 